\title{
La Politique du Bulldozer ou la Gestion Foncière au Cour des Tensions Sociales à Bamako au Mali
}

\author{
Dr. Mahamar Attino, PhD
}

Enseignant-Chercheur, à l'Institut de Pédagogie Universitaire (I.P.U), Bamako, Mali

Doi:10.19044/esj.2020.v16n29p266 URL:http://dx.doi.org/10.19044/esj.2020.v16n29p266

\section{Résumé}

Les difficultés inhérentes à l'accès à la propriété du sol amènent ces dernières décennies (1990-2010) les populations aux revenus modestes à occuper illégalement les espaces publics. Les autorités, pour libérer ces dits espaces, ont recours à la « politique du bulldozer » qui consiste à démolir par la force publique leurs propriétés bâties, ce qui envenime les tensions sociales autour du foncier urbain. À cet égard, l'objectif de cette étude empirique est d'apprécier l'utilisation du bulldozer comme outil de gestion par contrainte, et par ricochet d'en dégager ses répercussions sur le grignotage de l'espace intra- urbain de Bamako, la capitale du Mali. La méthodologie utilisée a consisté en la revue documentaire pour avoir un large spectre sur la question foncière en général et celle du foncier urbain à Bamako en particulier. Ensuite des outils d'enquête dont le questionnaire a été utilisé pour recueillir les données quantitatives, et un guide d'entretien pour glaner les informations qualitatives. Ces données recueillies ont été analysées, interprétées pour les besoins de la cause. Les principales trouvailles de ce travail sont entre autres : la découverte de l'informel comme moteur d'une économie urbaine ; l'épuisement presque total des réserves foncières urbaines ; la précarité des zones à risque où se déroule une violence économique et sociale et le désarroi des pouvoirs publics qui recourent de plus en plus à la politique du bulldozer pour déguerpir les occupations illicites des sites d'utilité publique. Il est de poids de rappeler que le Mali est un pays de l'Afrique Occidentale avec une superficie de $1.241 .238 \mathrm{~km} 2$, et une densité de 6 habitants $/ \mathrm{km} 2$ pour une population d'environ 2000.000 d'habitants.

Mots clés : Politique, Bulldozer, gestion foncière, tensions sociales, Bamako 


\title{
The Bulldozer Policy or Land Management at the Heart of Social Tensions in Bamako/Mali
}

\author{
Dr. Mahamar Attino, PhD
}

Teacher-Researcher at Institute of University Pedagogy, Bamako, Mali

\begin{abstract}
In recent decades (1990-2010), the difficulties inherent in accessing land ownership have led low-income populations to illegally occupy public spaces. The authorities, in a bid to free these so-called spaces, have recourse to the "bulldozer policy". This policy involves demolishing built properties by public force, which has exacerbated social tensions around urban land. In this regard, the aim of this empirical study is to assess the use of the bulldozer as a management tool by constraint. By extension, it aims to identify its repercussions on the nibbling of the intra-urban space of Bamako, the capital of Mali. The methodology used consisted of the documentary review to obtain a broad spectrum of the land issue in general and that of urban land in Bamako in particular. After then, survey tools including the questionnaire were used to collect quantitative data, and an interview guide was administered to glean qualitative information. All data collected were analyzed and interpreted for the purposes of the study. The main findings of this study are among others: the discovery of the informal as the motor of an urban economy; the almost total depletion of urban land reserves; the precariousness of risk areas where economic and social violence takes place; and the disarray of the public authorities which resort more and more to the policy of the bulldozer to clear away the illicit occupations of the sites of public utility.
\end{abstract}

Keywords: Policy, Bulldozer, Land Management, Social tensions, Bamako

\section{Introduction}

Le monde connait une croissance urbaine beaucoup plus importante (auteur ?). En effet, la population mondiale s'urbanise avec un rythme croissant. Selon les Nations Unies, la population urbaine mondiale avait atteint le seuil de $50 \%$ en 2007 dont 2 /3 vivent dans les pays en développement. Elle devrait connaitre une évolution significative dans les prochaines décennies en passant de $50 \%$ à $61 \%$ à l'horizon 2030 (Diakité, 2019, p.6).

En Afrique, un africain sur trois vivait en ville en 2000. Ce taux atteindra un sur deux en 2030 (Ajay-Kumar \& Fanny Barret, 2008). L'ONU- 
Habitat ajoute que si les taux d'accroissement urbain se maintiennent, plus de la moitié de la population en Afrique sera urbaine en 2050. Quant au Mali, la population urbaine est passée de $11 \%$ de la population totale en 1960 à $26 \%$ en 1994,28\% en 1996 et 30\% en 2000.En 2009,1 malien sur 3 vivait en ville et ce taux atteindra 1 sur 2 en 2024 (DNSI, 2009).

La ville de Bamako connait la même dynamique démographique et spatiale. Entre 1998 et 2009, le taux de croissance démographique était de $5,4 \%$, soit une population de 1.016.296 habitants en 1998 à 1.810.366 habitants en 2009 (RGPH, 2009).La population de Bamako est estimée à plus de 2.000.000 d'habitants. Or à l'horizon 2025, plus de 3.000.000 d'habitants résideront dans l'aire urbaine de Bamako (Mairie du District, Forum sur le développement urbain, 2010). L'évolution démographique a été consécutive avec l'expansion spatiale. Ainsi, le tissu urbain de Bamako est passé de 1200 ha en 1960 à 12000 ha en 1996 (Diarra, B et al., 2003). Actuellement, les chiffres parlent de 24000 ha (Forum de Bamako, 2010). Cette croissance démographique exige un besoin énorme d'espace, ce qui se traduit par une forte pression exercée sur les terrains urbains qui sont de plus en plus chers.

Pour toutes ces raisons, la question de l'accès à l'habitat ou à la propriété foncière se pose alors avec acquitté. La pression sur le sol urbain devient de plus en plus forte. Devant les besoins de plus en plus croissants de parcelles à usage d'habitation, les autorités procèdent à des lotissements planifiés. Mais c'est un secret de polichinelle qu'au Mali, pour être propriétaire il faut appartenir à la classe moyenne. C'est pourquoi les plus nantis ont décidé de faire une razzia sur les terrains urbains et périphériques, le plus souvent de manière illégale (ex : affaire des terrains de Tabacoro) (HTTPS /Koulouba .com/spéculation foncière).

L'accès à la propriété foncière est devenu un enjeu majeur dans le District de Bamako et par ricochet une source de tensions sociales permanentes. En effet, les conflits liés à la propriété foncière en milieu urbain impliquent plusieurs groupes d'acteurs. Il s'agit principalement des agents de l'administration publique, des propriétaires fonciers ou responsables coutumiers, les acquéreurs et les intermédiaires.

Cependant, il convient de préciser le fait que ces différents acteurs, même s'ils entretiennent des relations commerciales marchandes liées essentiellement à la rente foncière, sont en situation conflictuelle de façon permanente du fait de la divergence de leurs intérêts. À ceci il faut ajouter la méconnaissance de certains acteurs qui ignorent les limites des terres appartenant à l'État, surtout les autorités de tutelle. Les maires connaissent parfaitement les limites du domaine de l'État et du privé à travers de propriété étatique, mais n'hésitent généralement pas à vendre ou à morceler l'un comme l'autre. De manière générale, la vente des parcelles est monnaie courante au niveau des Communes de Bamako sauf celles qui ne possèdent plus de 
réserves foncières. Dans ce cas de figure, les conflits fonciers sont liés aux parcelles non construites. Ces terrains, une fois localisés, peuvent être saisis par la Mairie ou vendus par les spéculateurs fonciers et ce n'est que bien plus tard que le propriétaire l'apprend car il est généralement le dernier informé.

Une autre évidence est que les conflits fonciers sont surtout importants dans les quartiers périphériques où les responsables coutumiers et les démarcheurs (coxers) sont très actifs avec la complicité des agents de l'administration publique qui établissent moyennant redevances ou bakchichs de faux titres de propriété, objets de nombreux litiges.

Sur le plan social, l'importance de l'informel dans le marché foncier urbain a accéléré la montée du prix des parcelles, ce qui a pour conséquence directe l'exclusion des couches populaires de la propriété immobilière foncière par les filières formelles, d'où la multiplication des quartiers informels (épanouissement du spontané) dans le District de Bamako. Mais cela ne saurait signifier que la création et le développement des quartiers informels ne sont pas l'œuvre des seules couches populaires mais de nombreux acteurs parmi lesquels les élus locaux, les propriétaires coutumiers, les migrants maliens et même parfois des migrants étrangers adossés à des maliens.

$\mathrm{Au}$ niveau des structures officielles, le transfert de compétences dans le domaine foncier n'est pas encore en adéquation avec la loi de décentralisation. L'État central à présent a encore la main mise sur ce secteur si stratégique dans les centres urbains.

L'occupation des poumons d'aération, des places de loisir et de détente, des espaces verts est attestée dans presque toutes les Communes du district de Bamako.

Il est important de noter qu'aucune des six municipalités n'échappe en réalité à cet imbroglio que suscite les démembrements en catimini des espaces publics intra-urbains : ces grignotages d'espaces considérés «verts » et de terrains déjà réservés aux besoins communautaires, ces détournements collectifs que l'État semble renoncer à assumer (Traoré, 2019, p.274).

Bamako, aujourd'hui avec une population en constante évolution, connait de vives tensions d'appropriation et d'usage, non seulement sur les espaces publics du district, mais aussi sur les réserves foncières des communautés coutumières et des Communes rurales nouvellement constituées sur les périphéries urbaines (Traoré, 2019, p.274).

Les réserves foncières sont sous tension accrue, ce qui entraine une gouvernance contestée et une compétition de plus en plus âpre pour leur possession et leur gestion.

C'est ainsi que dans les années 2000, les tensions sociales grimpent ave la montée en force des violences physiques et des remous politiques sur les cas de «prédation» explicitement liés à l'appropriation disputée de 
terrains urbains et périurbains. Ces terrains achevés ou en chantier, les démolitions seront ordonnées par soit des individus ayant ester en justice, ou par décisions administratives pour cause d'utilité publique. Toujours est-il qu'il débouche sur des grincements de dents des suites de l'intervention du bulldozer. Une opération de ce genre plus mémorable est en cours (Février, 2020) sur le site des logements sociaux de Tabacoro où les occupants sont sommés de déguerpir pour la construction de logements sociaux (heurts et protestations ayant abouti à l'arrestation d'un prêcheur sulfureux pour outrage à autorité).

C'est une lapalissade que de dire que la gestion foncière au niveau de notre zone d'étude fait intervenir une myriade d'acteurs dont les rôles souvent se chevauchent et où au lieu d'avoir de la complémentarité, on assiste plutôt à un enchevêtrement dont le règlement n'est pas aisé. En effet le rôle des chefferies traditionnelles dans l'occupation foncière à Bamako a été décrié maintes fois. Les autorités coutumières avaient l'habitude de distribuer la terre au demandeur moyennant une redevance symbolique comme des noix de cola. Pour ces chefferies traditionnelles la terre a une valeur psychologique et appartient à la communauté qui peut la prêter ou la céder à autrui mais pas moyennant argent car pour elles donner une valeur marchande à la terre c'est la «désacraliser ». Aussi les autorités traditionnelles ont du mal jusqu'ici à comprendre qu'elles ne sont plus les seuls acteurs de la gestion foncière, et qu'elles doivent composer avec l'Etat a qui appartient la terre, le sol et le soussol du pays !

Quant aux institutions étatiques leur rôle dans la gestion foncière est complexe. Elles doivent réguler ; règlementer, sécuriser, délimiter les réserves foncières de l'Etat. Elles ont aussi pour mission de veiller à l'application stricte du contenu des documents de planification urbaine notamment le schéma directeur d'urbanisme, et les Plans d'urbanisme sectoriel : SDU et PUS. Également les services techniques doivent assurer la bonne tenue du cadastre et la sécurisation de la propriété foncière.

Le rôle de l'élite locale est versatile car elle oscille en fonction de ses intérêts. L'élite est la vraie détentrice des ficelles du désastre foncier urbain. Elle est souvent juge et parties. L'élite fait partie de la nomenclatura qui peut acheter plus de parcelles mais aussi elle peut acheter celles qui sont plus chères qu'elle peut revendre plus tard entretenant la spéculation foncière.

En cas de conflit entre acteurs du foncier la médiation est assurée par les institutions étatiques comme les commissaires-priseurs, les huissiers de justice, les commissariats de police, les tribunaux, la chambre de commerce,etc.

Les acteurs de la gouvernance locale en matière de gestion foncière sont de plus en plus confrontés au problème de l'occupation illégale de l'espace public par certains résidents urbains, soit pour y habiter, soit pour y 
mener des activités lucratives. C'est ainsi qu'à Bamako, face à l'occupation anarchique de l'espace public urbain par les populations avides de logements ou d'infrastructures commerciales, les autorités recourent ces dernières décennies (1990-2010) à la politique du bulldozer pour les faire déguerpir manu-militari.

C'est cette tactique sinon cette méthode pédagogique spécifique qui consiste à utiliser le bulldozer (engin de terrassement) pour démolir par la force publique les édifices bâtis sans autorisation, et généralement pour des raisons d'utilité publique, qui a été appelée « la politique du bulldozer », étant le recours à cet engin synonyme de contrainte et de coercition la problématique sur laquelle ce papier est centré.

Pour réussir cette étude des questions de recherche ont été élaborées, des objectifs ont été définis, et des hypothèses ont été formulées :

La question centrale est : le recours à la politique du bulldozer par les autorités ne traduit-il- pas l'échec de la gouvernance foncière en milieu urbain?

Cette question principale est étayée par des questions secondaires qui sont :

quels sont les effets de la politique du bulldozer sur l'occupation de

l'espace urbain à Bamako ?

_cette méthode pédagogique est-elle dissuasive et quelles en sont ses limites ?

_ y-a-t-il une alternative à cette politique ?

En ce qui concerne les objectifs de ce papier, l'objectif principal est d'étudier les effets de la politique du bulldozer sur l'occupation de l'espace public urbain et par ricochet d'étudier les péripéties de l'accès à la propriété du sol en milieu urbain et ses enjeux.

Les objectifs secondaires sont :

étudier les effets de la politique du bulldozer sur l'occupation de l'espace public urbain à Bamako ;

_ présenter ses limites à travers les enjeux et les péripéties de l'accès à la propriété du sol et ses retombées ;

_ proposer une alternative pour une meilleure gestion de la question foncière à Bamako.

Afin de parvenir à des résultats, un certain nombre d'hypothèses a été émis, étant l'hypothèse principale : le recours à la politique du bulldozer par les gouvernants traduit l'échec des politiques foncières urbaines. Les hypothèses secondaires s'articulent autour des réflexions suivantes :

_ les contraintes liées à l'accès à la propriété du sol en milieu urbain

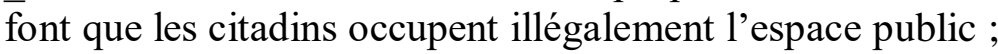


_la politique du bulldozer est une solution non pérenne et crée plus de problèmes qu'elle n'en résout ;

_respecter scrupuleusement le contenu des documents de la planification urbaine (SDU et PUS) peut être une alternative à cette politique.

Pour mener à bien cette étude, dans une première entrée les matériels et méthodes(méthodologie) sont présentés, dans la seconde entrée les principaux résultats sont donnés et puis dans la troisième entrée ces dits résultats sont analysés et discutés.

\section{Méthodologie}

Pour réussir cette étude empirique, la méthodologie est basée sur la revue critique de la littérature qui a permis de faire l'état des lieux de la recherche sur le foncier en général et l'accès à la propriété foncière en milieu urbain et les diverses controverses dont elle est à l'origine à la fois pour les pouvoirs publics que pour les résidents urbains et ceci urbi et orbi. La méthodologie choisie a aussi permis de choisir le type d'enquête à conduire pour les besoins de l'étude afin de collecter les données primaires nécessaires. Ainsi la méthode mixte avec une composante quantitative et une composante qualitative fut choisie, compte tenu des besoins en information.

L'enquête par questionnaire s'est déroulée dans la zone d'étude. Il comporte les axes suivants : Identification des enquêtés, titres de propriété, profession, degré d'implication dans la rente foncière, les coûts, les retombées de la spéculation, etc.

Quant à l'administration du guide d'entretien, elle a été individuelle et a été faite dans un endroit convenu en vue de garantir la confidentialité des informations obtenues auprès des personnes ressources.

L'échantillon de l'étude a porté sur 100 personnes choisie aléatoirement dont 90 répondants constitués de déguerpis des voies publiques, de propriétaires de maisons victimes de démolition, de locataires, de commerçants, de promoteurs immobiliers, de coxers (démarcheurs), de revendeurs. Quant à l'échantillon qualitatif (10 répondants) il est essentiellement constitué de responsables locaux des services techniques impliqués dans la gestion du foncier (maires, conseillers, agents des domaines et du cadastre, services des impôts, agents de sécurité, géomètres).

La méthode aléatoire a été utilisée en ce qui concerne le choix des populations cibles selon le sexe et la fonction (hommes, femmes, jeunes) à interviewer donc pour l'échantillon quantitatif.

Il convient également de préciser que toutes ces phases décrites cidessus ont été précédées par l'observation sur le terrain qui a permis de faire un état des lieux. 
Les théories qui sous-tendent cette étude sont celles de l'accès à la propriété du sol en milieu urbain, celle de l'acteur stratégique en sociologie des organisations développée par Michel Crozier (1970), mais aussi celle du changement social en Anthropologie défendue par Guy Rocher (1968), et celle de la gouvernance dans un contexte international de globalisation/ mondialisation, et celui actuel de décentralisation voire de régionalisation au Mali.

\section{Résultats}

Les principaux résultats de cette production indiquent principalement que les réserves foncières sont sous tension car leur gouvernance est contestée par des acteurs aux intérêts non convergents. Ils montrent aussi que la spéculation foncière est le moteur d'une économie urbaine florissante qu'on n'a pas encore fini d'étudier. Une autre trouvaille de taille est l'épuisement presque complet des réserves domaniales du périmètre urbain, ce qui oblige Bamako à lorgner du côté de ses voisins immédiats. In fine, cette étude indique également que les zones à risque sont le théâtre d'une violence à la fois économique mais aussi sociale du fait des tensions engendrées par l'imbroglio foncier qui multiplie les mécontents, lesquels constituent une bombe en léthargie dont l'explosion n'est pas toujours prévisible. L'ensemble de ces résultats sont déclinés comme suit :

\subsection{Présentation et analyse des résultats}

\subsubsection{Le marché foncier est au cœur de l'économie urbaine}

Il est d'importance de noter que le marché foncier bamakois est marqué par une opacité presque totale avec l'intervention d'une pléiade d'acteurs agissant en fonction de leurs moyens financiers, ou de l'efficacité de leur réseau social épars, ou de leur statut social. Les acteurs qui animent le marché foncier sont formels (ACI, Agences immobilières) et informels (les plus nombreux).

Dans ce marché foncier informel, certains acteurs jouent un rôle de relais entre les acheteurs et les vendeurs ou revendeurs de parcelles à usage d'habitation, industriel ou commercial. Ces intermédiaires procèdent par une augmentation du prix à chaque étape, ce qui entraine une surenchère active du prix du sol urbain. Les enquêtes sur le terrain ont permis de comprendre que le prix d'une parcelle dépend surtout de la « tête » du client, sinon par rapport au prix de cession des parcelles sont fixés comme suit selon la Mairie centrale :

_en zone commerciale, $1500 \mathrm{~F}$ le mètre carré ;

_en zone industrielle, $340 \mathrm{~F}$ le mètre carré ;

_en zone résidentielle, $300 \mathrm{~F}$ le mètre carré. 
Le réel constat est que le marché foncier à Bamako est organisé autour de réseaux bien structurés qui font vivre toute une économie urbaine souterraine. Aussi le caractère florissant du marché foncier a favorisé l'émergence d'un secteur informel très puissant qui est celui des démarcheurs ou « coxers » qui sont au cœur des transactions foncières et immobilières. En plus de l'acquisition à la construction de la propriété foncière ou immobilière, ils sont mobilisés et restent partie prenante voire incontournables. La prise en compte de leur rôle est déterminante pour comprendre les contours de la dynamique de ce marché.

Le dynamisme du marché foncier suivant les lois de l'offre et de la demande, a rapidement enclenché une activité de spéculation intense, ce qui a engendré en un temps deux mouvements (en un laps de temps). Une augmentation du prix le long du processus de vente.

Selon certaines statistiques récentes, 50 à 60\% (Deyoko, 1991, pp. 198-212) des parcelles attribuées sont revendues par leur propriétaire pour des raisons économiques. A Badalabougou, $60 \%$ des concessions construites en matériaux durables sont des concessions qui ont été vendues par leur propriétaire initial (idem, 1991, pp 198-212).

Un autre type de spéculation est lié aux personnes qui achètent des parcelles dans les quartiers lointains pour les revendre plus chères. Le prix de la parcelle, il convient de le rappeler, dépend de la tête de l'acquéreur ; c'est dans ce sens que le revendeur de la parcelle sait bien avec quel type de client il peut tirer davantage de profit. Le prix du terrain peut aussi dépendre de sa localisation géographique (distance par rapport au centre-ville, viabilisation, accès facile ou non aux artères principales). Il peut découler aussi de la structure du sol (facilite ou non à construire), de la proximité ou non des voies de communication.

Dans le but de rentabiliser les infrastructures commerciales urbaines tout en libérant les espaces publics entre Avril et Mai 2016 par les «opérations Ami Kane », les autorités urbaines organisèrent un déguerpissement le long des voies publiques. Bien qu'il soit un acte hautement nécessaire, il a fait beaucoup d'aigris et de mécontents (111000 personnes restées en chômage selon un recensement de la Mairie de la commune VI, 2016).

Le dimanche 17 Mars 2019 en commune V à Baco Djicoroni ACI, le Ministère de l'habitat a procédé à la destruction de certaines réalisations jugées illicites sur deux espaces. Ces activités rentrent dans le cadre du grand projet de sécurisation et de valorisation des espaces publics à Bamako. 
Carte 1. Bamako dans le Mali et le Mali en Afrique

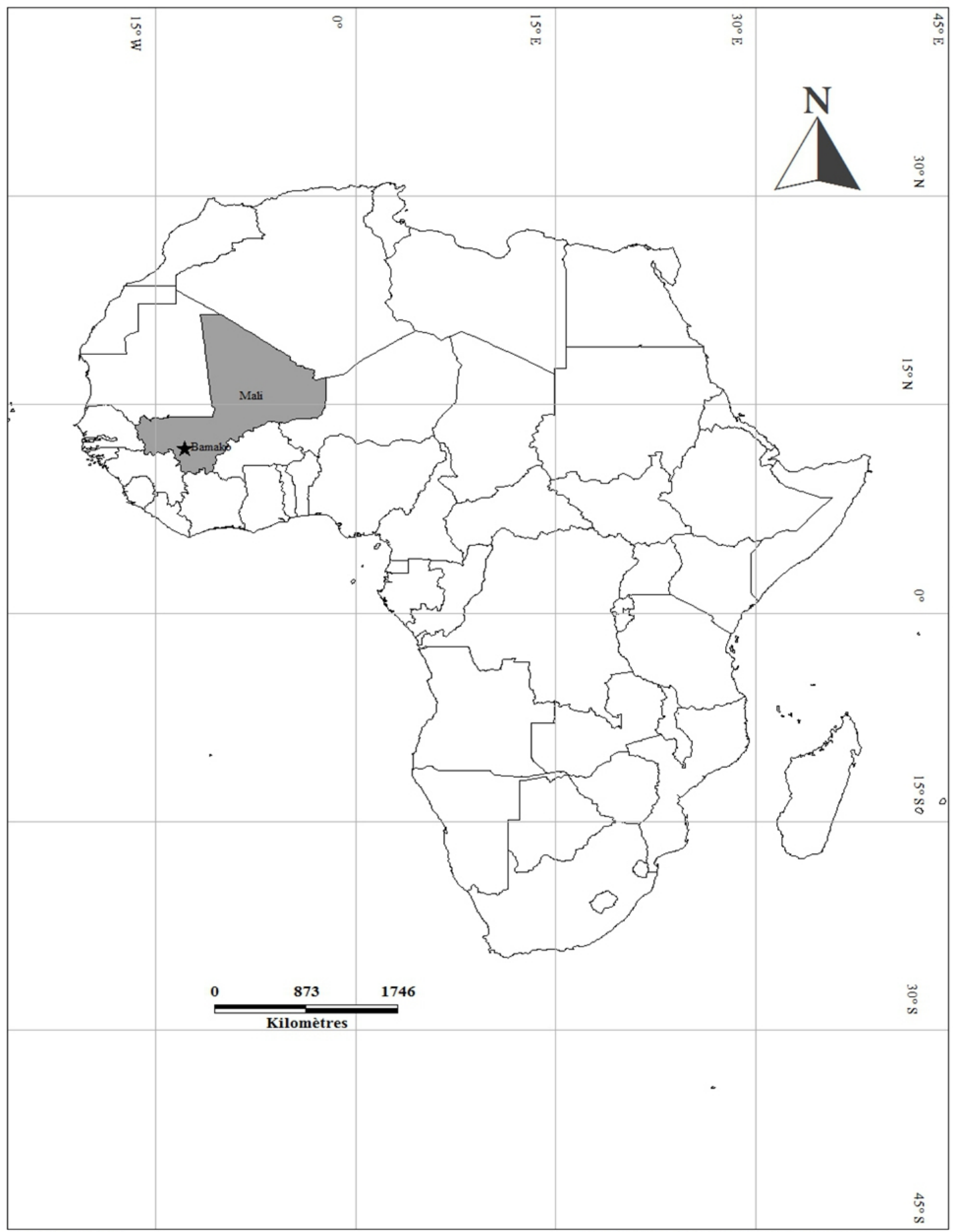




\section{1.2. L'épuisement du restant des réserves domaniales}

La pression croissante et constante par la forte demande conduit à la diminution voire à l'épuisement presque complet des réserves foncières des Communes de la rive gauche du fleuve Niger (Commune I, II III et IV) depuis les années 1980. Les réserves des Communes de la rive droite du Niger (Communes V et VI) sont en voie d'épuisement, et de fait elles jettent leur dévolu sur les réserves des Communes rurales avoisinantes comme celle de N'Gabakoro droit, de Mountougoula, celles de Kalabancoro, celles du Mandé, etc. Ceci d'autant plus que les années 1990 ont vu l'État procéder à des arbitrages publics en matière de litiges fonciers. Les débuts de la III -ème République ont vu non seulement la décentralisation programmée au niveau national, mais aussi de sensibles mutations des gestionnaires qui ont fait s'envoler le prix des parcelles à bâtir dans la capitale, Bamako (Bertrand, 2003). Le prix de la parcelle a évolué de 3.000.000 de francs dans les années 1990 pour une superficie de $400 \mathrm{~m}^{2}$ à 12.000 .000 de francs à partir de 2009. Concomitamment à cette montée en flèche de la valeur vénale du sol urbain, les dimensions des parcelles à bâtir vont diminuer $\left(300 \mathrm{~m}^{2}\right)$.

C'est dans cette mouvance spéculative que l'État à travers l'ACI (Agence de Cession Immobilière) va procéder au morcellement des dernières importantes réserves foncières de Bamako pour les vendre aux enchères publiques au plus offrant et au dernier enchérisseur. À partir de là, les transactions se focalisent désormais sur la valeur $\mathrm{du}^{2}$ de terrain tout en tenant toujours compte de sa localisation et surtout de son degré de viabilisation.

La différence fondamentale entre l'ACI et les lotissements administratifs destinés à la réalisation de concessions à usage d'habitation, est que l'ACI procède à l'achat et surtout à l'établissement de titres hypothécables, ce qui implique une valorisation assurée à la valeur domaniale.

Ainsi, les cessions opérées par l'ACI sur le domaine de l'État seront à l'origine d'une forte diffusion de titres de propriété dits définitifs (TF) de la propriété résidentielle, documents d'autant plus onéreux qu'ils sont susceptibles de servir de garantie (monnaie d'échange) pour les prêts bancaires.

Le RGPHP de 2009, a estimé qu'il y avait plus de 56.000 ménages titulaires d'un titre foncier, soit $11 \%$ des modes d'occupation du logement dans le District de Bamako. Par ailleurs, 39\% des ménages n'ont pas de titres de propriété régulière ou sont titulaires de simples permis d'habiter, et d'usufruits familiaux. Avec l'ACI, les classes sociales émergeantes ont un engouement marqué pour les références écrites plus facilement conservées et qui sont garants d'une propriété individualisée faisant oublier les tracasseries des grandes familles traditionnelles de Bamako. 
D'autre part, suite à la mauvaise gestion du patrimoine foncier et l'occupation anarchique des espaces publics, des mesures idoines furent préconisées par les services techniques : notamment faire une relecture des textes, auditer le foncier, immatriculer tout le domaine public, établir une SIF (système d'information foncier), sanctionner les spéculateurs. Ceci est d'autant plus vrai que les litiges fonciers sont liés à la faiblesse du code domanial et foncier, à la réglementation foncière et à la spéculation foncière.

Tableau 1. Réserves foncières de Bamako.

\begin{tabular}{cccc}
\hline Numéro & Commune & Localisation & Superficie en ha \\
\hline 1 & Sanankoroba & Banankoro & 50 \\
2 & Safo & Safo & 100 \\
3 & Mountougoula & Mountougoula & 200 \\
4 & Kalabancoro & Diatoula & 300 \\
5 & Ngabakoro_Droit & Djinkoni & 200 \\
6 & Mandé & Mandé & 50 \\
\hline Total & & & 900ha \\
\hline
\end{tabular}

Source : SDU de Bamako et environ, $3^{\mathrm{e}}$ révision, 1996.

Bien que ces réserves soient d'importance variable, elles ne peuvent plus satisfaire la demande toujours croissante de la population urbaine. Elles sont aussi toutes localisées dans l'hinterland de la ville. L'exemple le plus patent est celui de Kalabancoro qui a vu toutes ses réserves vendues en totalité (300ha).

Graphique 1. Réserves foncières de Bamako

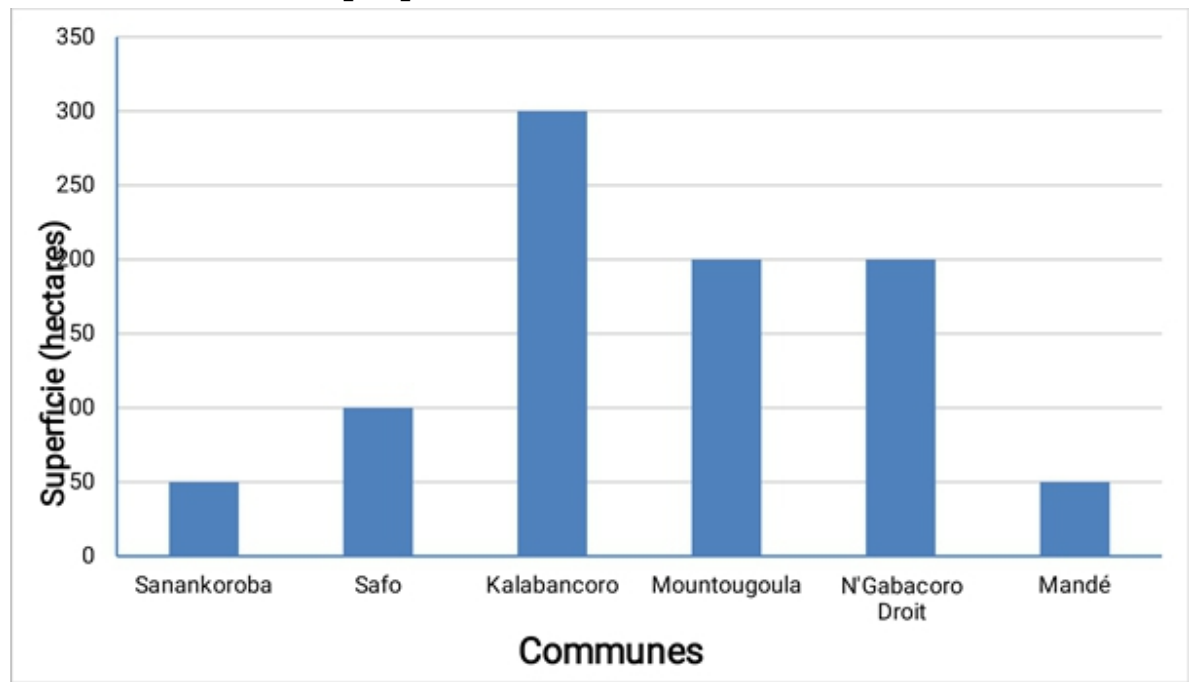

Source : Enquêtes , 2020.

Le graphique montre que les réserves importantes se localisent dans les communes avoisinantes. Les plus visibles sont celles de Kalabancoro, puis 
viennent celles de Mountougoula et de N'Gabakoro toutes situées dans le Cercle de Kati jouxtant le District de Bamako.

\subsubsection{Les zones à risque sont le théâtre de violences économique et sociale}

Les visionnaires ayant suivi leur sixième sens ont observé avec attention l'extension progressive lente mais sure de la ville de Bamako sur la rive droite à partir de années 1970. Ayant constamment à l'esprit que le futur du pays est et sera à Bamako, une poignée de visionnaires a commencé à acheter des terres de plus en plus loin de la périphérie immédiate dans le but d'y établir des vergers, des jardins sinon des « foro » qui voient la ville se vider de ces résidents fortunés toutes les fins de semaine pour échapper au tumulte, aux nuisances, et à la chaleur suffocante d'une ville de plus en plus pleine à exploser. Ces nouvelles acquisitions de parcelles deviennent des concessions rurales qui plus tard ont été morcelées et vendues aux nouvelles classes moyennes dont le «night mare » demeure l'accès à la propriété du sol en guise de résidence. Les nouvelles possessions domaniales sont généralement transformées en lieu de pratique d'aviculture, de mangueraies, ou de parcs à stabulation pour leur troupeau de bovins. Ils y creusent la plupart du temps un puits à grand diamètre, et y recrutent un gardien des lieux qui s'y installe avec sa maisonnée. Ceci va en droite ligne du constat dressé par l'analyse de Bertrand (2015) dans «Du District au grand Bamako (Mali): réserves foncières, extension, gouvernance contestée. ».

À partir des années 2000, ces propriétaires terriens ont commencé à morceler leurs champs qu'ils ont garanti au paravent en acquérant un titre définitif « $\mathrm{TF}$ » leur garantissant ad aeternam la possession définitive. La dimension moyenne de ces champs urbains est de 5 ha même si les nantis achètent et juxtaposent deux ou trois parcelles contigües de même dimension. L'étude de ce phénomène est bien rendue dans le papier de A Keita (2003) dans le cas du phénomène des citadins paysans au Mali où l'auteur s'interroge s'il s'agit d'une stratégie de création d'exploitations agricole moderne ou de spéculation foncière tout court. La violence apparait dans les cas où les titres définitifs ne sont pas acquis, ou quand il y a superposition de titres de propriété sur la même parcelle. Également, ce sont les héritiers des propriétaires initiaux de ces « champs » qui vont faire face aux nouveaux spéculateurs terriens et immobiliers qui se présentent aux ayants droit avec des titres obtenus avec la complicité de certains agents véreux des services de la régulation foncière ou par la complicité d'un membre vendu de la famille qui pour des raisons économiques ou autres ont vendu une ou des parcelles de la propriété collective à l'insu des autres ayants droit. Ainsi, les terres des périphéries urbaines de Bamako sont soumises à un tiraillement entre racines, épargnes et spéculations entrainant de nouvelles appropriations foncières et des recompositions de l'espace périurbain ceinturant Bamako comme pour 
paraphraser (Fautras, 2016). C'est souvent avec les sommations d'huissiers de justice ou des fois avec les levées topographiques de géomètres que ces propriétaires spoliés se rendent à l'évidence. Cette découverte surprenante amène des troubles psychologiques mais aussi des saignées monétaires car ils doivent désormais aller au tribunal pour répondre aux injonctions des nouveaux propriétaires et justifier leur titre de propriété. Les va et viens au tribunal, la valse entre les ordres des tribunaux engendrent une saignée financière mais aussi un éclatement du tissu familial sinon social en plus de la perte de temps et d'énergie. L'implication des agences immobilières privées et des banques en quête de terrains à usage d'habitation, en plus de l'État qui, à travers sa politique de logements "dits sociaux », a besoin de plus en plus de terres vacantes dans le pourtour immédiat du centre-ville entrainent de nouveaux déguerpissements entrainant des grincements de dents, de mécontents et de plus d'opérations "Ami Kane » où le bulldozer s'exprime généralement et accroit le nombre d'exclus ou de sans abri dans la ville de Bamako. Aussi, ces anciens champs vont assister à une reconversion sociale dans la mesure où au départ ils étaient conçus pour être des vergers voués à l'agriculture et de nos jours les nouveaux propriétaires changent leur vocation en concession à usage d'habitation qu'on rejoint à la périphérie après une rude journée de labeur et un trafic alternatif agaçant. À ce sujet, un chercheur a pu noter ceci : «mais tandis que la marchandisation brutale des domaines ancestraux se traduit par une spéculation foncière intense et par la privatisation illégale de l'espace public, la prolifération normative favorise le droit du plus riche et accroit encore la gravité des conflits fonciers » (Bouju, 2009).

Les démarcheurs appelés ici les «coxers » assurent l'intermédiation entre les individus et les institutions ou entre les responsables coutumiers et les acheteurs. Ils sont devenus incontournables et agissent au vu et au su de tout le monde, même si on sait qu'ils évoluent dans l'informel. Ils sont au centre d'un réseau clientéliste, affairiste, de courtisanerie qui met en relation le candidat ou demandeur et le responsable communal (maire, conseiller domanial) ou la chefferie traditionnelle du quartier pour la transaction financière. Mieux, on peut affirmer sans risque de se tromper que les magouilles ou malversations foncières s'organisent avec la complicité des officiels. Car dans la pratique, chaque maire s'attache les bonnes grâces des dignitaires du pays (président, ministre, députés, officiers supérieur, magistrats). Ainsi, à chaque morcellement les cadres politiques et administratifs sont servis en parcelles en guise de présents sur les réserves foncières de la Commune. Cette générosité non gratuite assure aux donateurs une protection de l'autorité centrale en cas de poursuites judiciaires (Bouju, 2004, p.148). Les maires quant à eux, pour des raisons mercantiles et électoralistes, se permettent d'attribuer des parcelles pour légitimer leur pouvoir en faisant des largesses à leur électorat. Ainsi, on peut noter que les 
édiles des grandes agglomérations depuis la création des Collectivités Territoriales (1990), continuent à vendre les parcelles sans l'autorisation préalable et règlementaire de l'autorité de tutelle (Gouverneur), l'essentiel des parcelles de terrains encore disponibles. Au niveau des structures officielles le transfert de compétences dans le domaine foncier n'est pas encore en adéquation avec la loi de la décentralisation. Un autre facteur de tensions sociales est le fait que dans la décentralisation les bailleurs de fonds exigent que l'État se retire comme instance de régulation en matière foncière, ce qui laisse le champ libre aux logiques marchandes voire affairistes ; c'est-à-dire, à des formes de régulation clientélistes.

Le 12 juin 2020, à quelques encablures du site de l'Université de Kabala, les autorités ont procédé à la démolition d'un ensemble de maisons bâties sur 10 ha situées sur un terrain appartenant à un premier propriétaire disposant d'un titre foncier en bonne et due forme.

Selon les enquêtes faites dans le District de Bamako en général, les conflits liés au foncier sont de deux types : la première catégorie concerne la superposition de titres de propriété sur la même parcelle. Dans ce cas de figure le conflit est provoqué entre les populations suite à certains actes administratifs attribuant à plusieurs personnes la même parcelle.

Quant à la seconde forme de conflit foncier, elle est celle née des opérations de viabilisation des quartiers spontanés ou périphériques. Ce genre de conflit oppose les autorités communales et les populations et se traduit le plus souvent par des rixes et heurts souvent violents. Les personnes dont les habitations sont affectées par ces opérations s'opposent vivement à leur déguerpissement, d'où l'intervention de bulldozers appuyés par les forces de l'ordre. Ainsi débute alors le hideux spectacle de jets de pierres et de grenades lacrymogènes entre la population se sentant abusée voire désabusée et les agents de sécurité mandatés pour le maintien d'ordre. Ce genre d'opérations a eu lieu dans plusieurs quartiers à partir des années 1991-2000. L'une des plus mémorables dates de 2008 s'est déroulée à Yirimadio et Missabougou en Commune VI. En effet, au cours de cette vigoureuse opération, plusieurs réalisations construites sur 95 ha ont été démolies par la Mairie centrale et le Gouvernorat qui pour y opérer les ont d'abord qualifiées de constructions anarchiques ou illicites.

Les conflits fonciers trouvent leur origine également dans l'existence de plusieurs structures de gestion mais aussi dans la cohabitation du droit moderne et du droit coutumier. Cette dualité ou du moins cette juxtaposition du droit moderne et du droit coutumier est pour beaucoup dans l'imbroglio au niveau domanial et foncier. En effet, les catégories du droit et l'irrégularité se brouillent au sein du district, car certains conflits opposent des occupants sans titres que la lutte contre la pauvreté encourage à s'exprimer, et deviennent les nouveaux occupants des places dites vacantes (Traoré, 2019, p. 273). 
Pour régler le problème de l'insécurité juridique et foncière relative à la superposition de titres sur une même parcelle, la primauté est généralement donnée au titre antérieur. Au cas contraire, la parcelle est attribuée à celui qui a réalisé des investissements lourds et sert de jurisprudence. On peut généraliser en notant que les conflits fonciers interviennent entre différents acteurs notamment :

_ entre la masse urbaine paupérisée et la classe moyenne ayant usurpé la plupart du temps certains privilèges de sa position administrative ;

_ souvent ce sont des conflits opposant la population contre la mairie comme lors des déguerpissements ou des opérations «Ami Kane » pour libérer les espaces publics et les trottoirs, ou lors des opérations de recasement et même de distribution des stands à usage commercial au grand marché ;

_souvent ces conflits opposent la population urbaine à l'Etat lui-même quand celui-ci veut transformer des anciennes concessions rurales à usage d'habitation, ou lorsque l'Etat procède à des déguerpissements pour des raisons d'utilité publique comme le tracé de la voirie, ou l'aération du tissu urbain parmi tant d'autres exemples.

Une autre évidence est que les conflits fonciers sont surtout importants dans les quartiers périphériques où les responsables coutumiers et les démarcheurs (coxers) sont très actifs avec la complicité des agents de l'administration publique qui établissent moyennant redevances ou bakchichs de faux titres de propriété, objets de nombreux litiges.

Aussi la mise sur le marché de terres appartenant à l'État ou relevant du domaine privé par les agents municipaux, s'explique en effet par le fait que ces agents aussi bien que les maires ne disposent pas d'autres sources de revenus que ceux procurés par la vente de parcelles la plupart du temps, car statutairement ils n'ont pas de salaires et n'ont droit qu'à des indemnités. C'est la raison pour laquelle à Bamako aujourd'hui, c'est une pratique courante de voir que les quelques terrains disponibles, les sites destinés hier au culte tels que les emplacements des mosquées, des églises, les terrains de football, les espaces verts ou toutes autres parcelles publiques ou privées sont généralement vendues par les maires, les propriétaires fonciers, les « coxers », sans se soucier des problèmes que cela peut poser en matière d'aménagement urbain et des tensions sociales sous-jacentes.

À travers les conflits fonciers dans le District de Bamako, on peut lire en réalité une politique tacite de laisser-faire de la part des autorités où chaque acteur opère dans son coin selon ses propres moyens et les opportunités qui se présentent à lui. Dans ce marché informel, certains acteurs jouent un rôle de relais entre les acheteurs et vendeurs ou revendeurs de parcelles tout en augmentant les prix à chaque niveau, ce qui donne lieu à un phénomène de surenchère active des prix du foncier (Keita, 2016, p.132). 
Carte 2. Les Communes soumises à de fortes pressions foncières

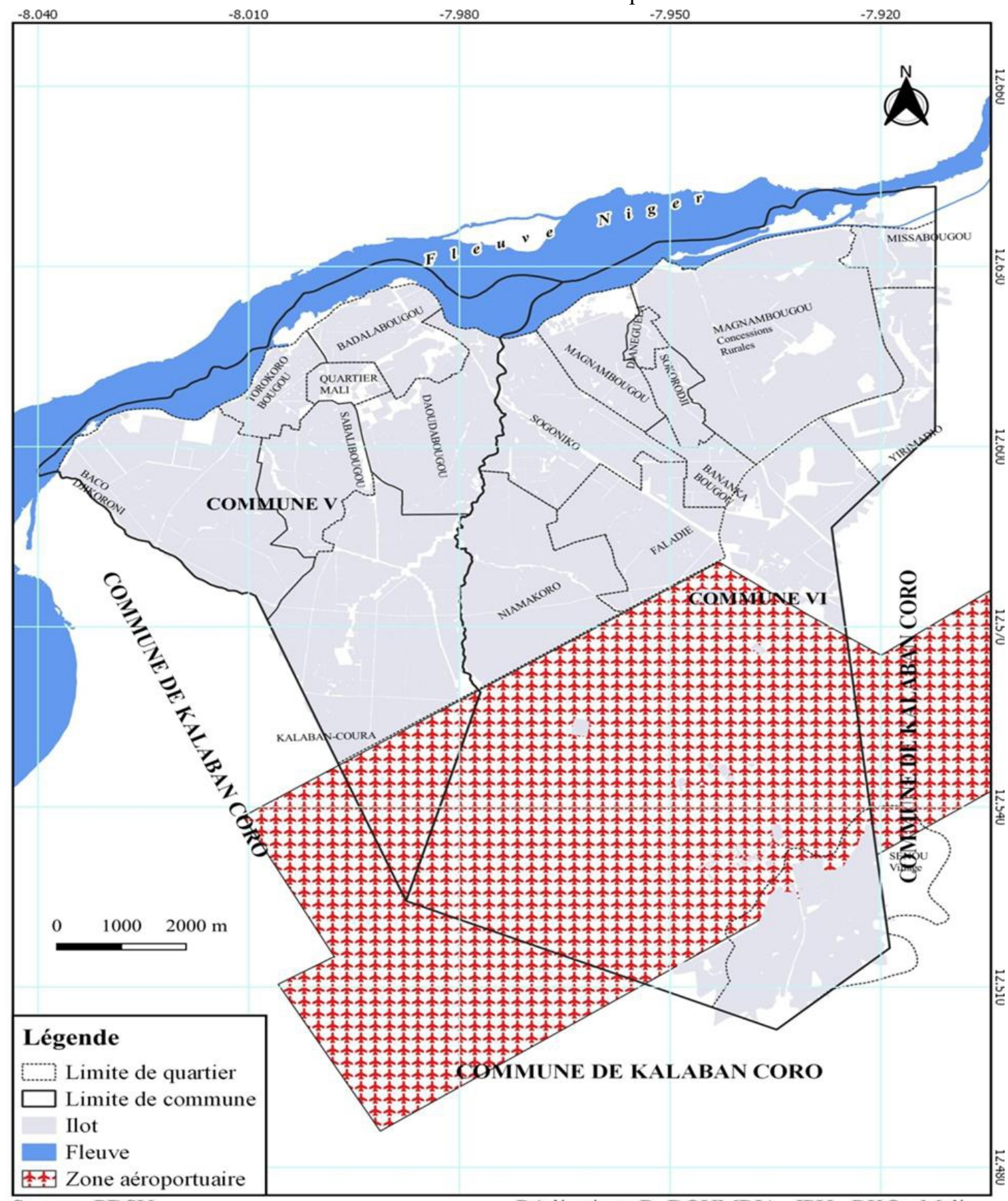

Source : PDSU. 
Carte 3. Les communes de Bamako

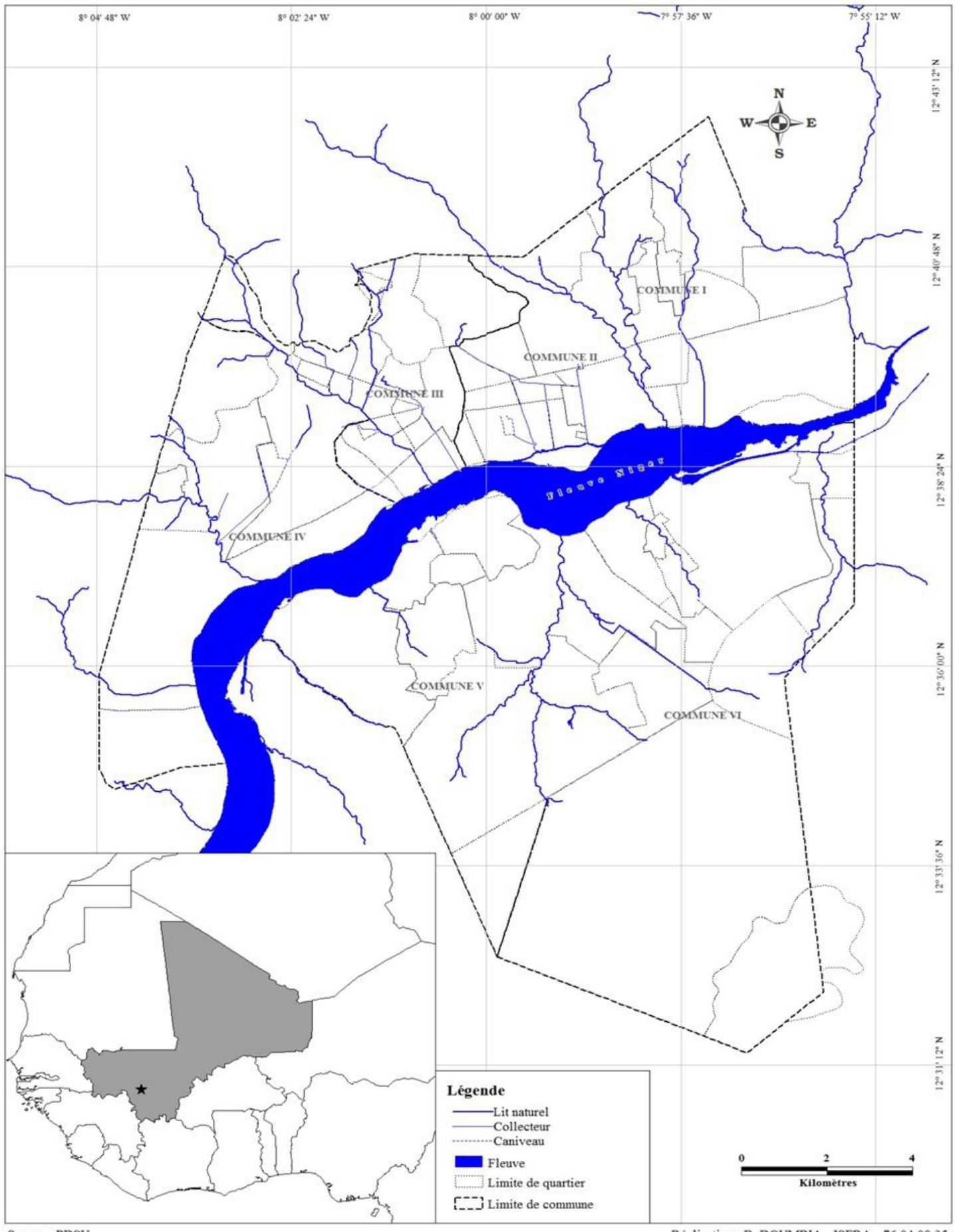

Source : PDSU

Réalisation : B. DOUMBIA - ISFRA - 76040835 
Tableau 2. Les différentes zones de tension en rive droite (Commune V et Commune VI).

\begin{tabular}{|c|c|c|}
\hline Lieux & Commune & Date \\
\hline Espace vert de Kalabancoro & Commune V & 2011 \\
\hline Espace vert de Daoudabougou & Commune V & 2012 \\
\hline Diatoula & $\begin{array}{c}\text { Sous-préfecture de Kalabancoro } \\
\text { vendue depuis senou }\end{array}$ & 2012 \\
\hline $\begin{array}{c}\text { Colline de Dougakoulou de } \\
\text { Yirimadio }\end{array}$ & Commune VI & 2011 \\
\hline Logements sociaux de yirimadio & Commune VI & 2011 2012 \\
\hline Site des logements de Tabacoro & Commune VI & 2020 \\
\hline $\begin{array}{c}\text { Niamakoro-Diallobougou-Sénou } \\
\text { Zone aéroportuaire (9000ha) } \\
\text { Niamakoro, Diallobougou, } \\
\text { Sénou. }\end{array}$ & 2019 \\
\hline $\begin{array}{c}\text { Litige foncier de } \\
\text { maisons furent démolies. }\end{array}$ & Commune VI & 28 février 2020 \\
\hline $\begin{array}{c}\text { Yirimadio Kouloubleni(place du } \\
\text { marché). }\end{array}$ & Commune VI & 10 mars 2020 \\
\hline
\end{tabular}

Source : Bertrand (2012)

La bombe foncière est en phase de devenir une bombe diplomatique si l'on se réfère à l'incident enclenché entre l'Egypte et le Mali lorsque l'Egypte a acheté auprès du gouvernement malien dans la zone du Mandé (Kangaba) 80 ha pour des fins agricoles. Mais le projet se trouve compromis aujourd'hui à cause de l'opposition des qui sans hésiter ont procédé au morcellement des parcelles sur lesquelles portent l'engagement du Mali auprès de la République sœur d'Egypte. Sur les 80 ha prévus seuls 15 ha sont actuellement disponibles, ce qui amena les partenaires à aller à Bewani en Zone Office du Niger $(\mathrm{ON})$.

Un autre cas illustratif et non des moindres est celui de l'interconnexion électrique entre le Mali et la Guinée portant une autre zone du Mandé (Badjoliba, Kangaba) où pour commencer les travaux pour une indemnisation des populations riveraines était prévue. Les populations une fois indemnisées ont refusé de libérer les lieux.

Un autre maillon de l'imbroglio foncier découle du problème de signature entre le maire et le sous-préfet à partir de 2008. En effet, le code domanial et foncier stipule que les sous-préfets ne peuvent pas donner des concessions à usage d'habitation, donc pour contourner la loi, les préfets délivrent des parelles entre 0 et 2,5 ha mais en parcelles à usage agropastoral. C'est ce qui est à la base du problème des déguerpis de Tabacoro pour l'implantation des logements sociaux dans cette zone. Même si certains logements sont censés être accessibles aux foyers peu fortunés, l'intense spéculation foncière et la bulle foncière dans les zones périurbaines a entrainé une rapide hausse du prix de ces parcelles (Franchette, 2011). 
Tableau 3. Évolution des espaces vides en ha de 1986 à 2000 en rive droite du fleuve à Bamako.

\begin{tabular}{lcc}
\hline \multicolumn{1}{c}{ Espaces vides } & 1986 & 2000 \\
\hline Badalabougou & 1127 & 241 \\
Niamakoro & 81 & 275 \\
Faso_Kanu & 109 & 67 \\
Banankabougou & & 72 \\
\hline Bako_Djikoroni & & 495 \\
\hline
\end{tabular}

Source : Traoré (2019, p.126)

On observe une diminution continue des espaces interstitiels entre les deux bornes chronologiques. Le tableau ci-dessous donne une idée sur l'évolution moyenne de l'espace en communes V et VI.

Tableau 4 : Evolution moyenne de l'espace en Communes V et VI de 1960 à 2016.

\begin{tabular}{lcc}
\hline Années & Pourcentages & Par / an \\
\hline $1960-1976$ & 1,2 & 16 \\
$1976-1998$ & 7,1 & 22 \\
\hline $1998-2016$ & 36 & 18 \\
\hline
\end{tabular}

Source : Traoré, 2019, p.126.

L'évolution de l'espace est moindre de 1960 à 1976. Elle devient importante dans les décennies 1990 à 2000 des suites de la pression démographique et du besoin croissant en espace à aménager. De façon générale, cette pression sur l'espace urbain est forte dans les Communes de la rive droite car elles sont presque les seules disposant encore d'espace aménageable sinon urbanisable.

En plus il convient de noter que l'analyse du contenu du discours des interviewés a permis de mieux mesurer l'ampleur, les retombées et le caractère sensible de l'ampliation de la politique du bulldozer sur les populations victimes. En effet, M.S a dit : «comment un pays normal peut déposséder ses citoyens et les jeter dans la rue sans aucune pitié. Peut-on bénir un tel État?». A.T, est commerçant et a été victime de l'opération dite «Ami Kane », "comment vais-je nourrir mes 13 enfants ? Le pays ne me donne plus le choix, je suis contraint d'être un truand. ». Y. K; déclare avec énergie: "Nous disposons de permis d'occupation délivré par la mairie du district de Bamako. Donc nous avons l'autorisation d'habiter ce site tout en brandissant son titre de propriété comme preuve à l'appui ». O.C, un agent d'une mairie, confirme l'octroi par l'administration de titres de propriété tout en ajoutant qu'ils savent que c'est illicite car ce sont des titres provisoires, mais au moment de conclure la vente les spéculateurs brandissent des autorisations d'occupation signées par la mairie du District de Bamako. .S.M ; coxer (démarcheur) affirme : "j'ai eu à acheter plusieurs parcelles à vil prix. On m'a dit de ne rien craindre car des personnalités du pays ont leurs parelles 
et ont des parcelles dans cette zone. ». M. D, chauffeur de son État qui fit partie des déguerpis de 1995 de la zone aéroportuaire des occupations illicites et anarchiques construites dans la zone aéroportuaire, ne cache pas sa colère : "la plupart des gens qui habitaient cette zone en Commune V avaient été recasés. Mais quelques années après ils ont réoccupé les mêmes parcelles pour lesquelles ils avaient été dédommagés. Ils ont fondé leur famille sur du faux». Et il ajoute : "c'est avec la complicité des maires et des chefs coutumiers que les gens continuent d'acheter des parcelles dans la zone aéroportuaire. ». Pour éviter que le scénario se répète, un agent attitré du service des domaines et du cadastre précise : «En début d'année de vastes opérations de démolition ont été prévues. Mais il a été demandé de surseoir aux opérations de démolition pour des raisons sociologiques. Les occupants ont été informés et les zones concernées ont été repérées. Les habitants ne seront pas surpris. ".

A. D. est géomètre ; il confirme : "la zone aéroportuaire est classée zone d'équipements et ne saurait être utilisée à d'autres fins sans l'autorisation expresse de l'Assemblée Nationale ». Pour des raisons d'utilité publique l'État doit annuler les titres de propriété attribués aux occupants pour libérer définitivement ladite zone.

Il ressort de l'analyse de ces discours que les différents acteurs ont tous un degré de responsabilité dans l'imbroglio foncier urbain. Chacun semble tirer « la couverture de son côté » mais il est évident que la responsabilité reste partagée. L'État pour réguler et faire en sorte que la force reste à la loi a recours à la tactique du bulldozer pour libérer par la force l'espace public, ce qui fait beaucoup de mécontents qui rejettent toujours la responsabilité sur l'Etat.

\section{Discussion des résultats}

L'accès à la propriété du sol en milieu urbain est un enjeu crucial pour tous les acteurs. En effet, d'accès libre jusque dans les années 1960-1970, la terre à laquelle on accédait juste après quelques conciliabules symboliques auprès des autorités coutumières, est devenue aujourd'hui un parcours de combattant pour les nouveaux citadins en quête de leur chez-soi. Si jadis la terre appartenait à la communauté et était gérée par un chef des terres, elle était une propriété collective familiale, et ne saurait souffrir de vente, car la vente la «désacralise». Mais la croissance démographique exponentielle et l'urbanisation galopante avec la macrocéphalie Cette demande croissante entraîne une prédation continue des espaces interstitiels intra-urbains constituant jadis des espaces verts et des lieux de loisir publics, mais qui de plus en plus sont phagocytés ou occupés illégalement par des acquéreurs disposant de titres de propriété la plupart du temps non définitifs. Leur désappropriation par la force publique pour raison d'utilité publique conduit 
généralement à l'entrée en scène du bulldozer comme instrument de résolution des tensions foncières mais qui en réalité crée de nouvelles tensions sociales avec son lot de déguerpis, de spoliés et de mécontents. Les resserves foncières sont en tension permanente de nos jours, ce qui entraine une contestation prononcée de la gouvernance domaniale par les autorités. Le grignotage continu des espaces interstitiels aboutit à l'usurpation sinon au détournement des réserves foncières des communes rurales constituées sur les périphéries urbaines de Bamako. L'accès aux terres périurbaines était " presque libre » jusqu'à une date récente. Mais les habitants et autorités coutumières de la périphérie se rendant de plus en plus compte que la terre a une valeur marchande, s'étant frottés aux autres acteurs, ont fini par comprendre que la terre est source de richesse monétaire dont la valeur augmente en fonction de sa localisation et de son degré de viabilisation. Les résultats de la présente étude corroborent ceux de beaucoup d'autres chercheurs qui reconnaissent que l'accès à la terre est devenu un enjeu majeur (Bouju, 2009 ; idem, 2004) (Deyoko, 1991).

L'une des trouvailles de cette étude est aussi que l'informel en matière d'accès à la propriété du sol urbain se trouve au cœur de l'économie urbaine en se référant aux transactions (vente, revente, location, cession) des différents acteurs et leurs retombées sur l'économie locale même si leur quantification reste à faire avec précision.

La superposition des types de droit coutumier et moderne est aussi une source non négligeable de conflits en matière foncière à Bamako. En effet, les catégories du droit et l'irrégularité se brouillent au sein du district, car certains conflits opposent des occupants sans titres, que la lutte contre la pauvreté encourage à s'exprimer, et les nouveaux occupants des places dites vacantes.

La pratique ou le recours à la politique du bulldozer n'est pas spécifique à Bamako, et est pratiquée ailleurs face au problème identique d'occupation anarchique ou non contrôlée de l'espace public (Dorléans, 1993 ; Loungou, 2004).

Quant à l'épuisement des réserves foncières, il est attesté ailleurs par certains auteurs (Jalabergt, 1971 ; Goislard, 1997 ; Aveline, 1995).

L'expression de la violence à la fois économique et sociale apparait de façon nette à travers les écrits de Choplin (2006), Bourdarias (1999), Fanchette (2011), et même Fatras (2016).

À la lumière de cette littérature, il est possible d'affirmer in fine que la spéculation foncière est un phénomène urbain dont l'acuité dépend de la rigueur des autorités chargées de la réguler et que la tactique ou pédagogie du bulldozer est une pratique courante à laquelle les gouvernants ont recours très souvent pour obliger les gouvernés à respecter l'espace public et à les imposer la civilité. 
Les facteurs qui sous-tendent les différentes tensions autour du foncier à Bamako sont entre autres :la dynamique foncière, qui est caractérisée par une spontanéité et une fragilité des mécanismes mis en place ; les documents d'urbanisme (SDU et PUS) qui bien qu'ils précisent les règles de servitudes d'occupation des sols ne sont pas respectées (les berges du fleuve, flans des marigots, servitudes des rails, etc.) ; l'ignorance des critères de recasement par la majorité des populations victimes, ce qui les écarte par conséquent des opérations de recasement. Le réflexe de ces populations déflatées, c'est de chercher à occuper des espaces non occupés même si ces lieux ne sont pas lotis ou répertoriés par les services publics. On peut également ajouter la forte demande de parcelles pour décongestionner les vieux quartiers centraux (Bagadadji, Niaréla, Dravela, etc.).

Il y a aussi le choc entre la « volonté affichée de l'État » à libérer les espaces et celle «intégrée des populations » de s'y opposer ou du moins de n'obtempérer qu'après le dédommagement. On peut y ajouter le fait que le Code Domanial et Foncier connait de réelles difficultés d'application dues soit à l'ignorance et au non-respect des lois foncières des acteurs en charge de la question foncière, soit à la mauvaise tenue du livre foncier ou cadastre rendant l'exploitation des archives foncières difficile, et l'insuffisance des outils de gestion foncière. L'insécurité foncière peut découler aussi du double emploi des titres fonciers (TF) à l'occupation anarchique des parcelles sans tenir compte des mesures d'urbanisme opérationnel.

Quant aux stratégies mobilisées par les différents acteurs pour se positionner sur le marché foncier, et les changements qui en découlent, on peut noter :

Le marché foncier de Bamako est caractérisé par une opacité totale avec l'intervention d'une pléiade d'acteurs agissant chacun en fonction de ses moyens financiers, de l'efficacité de son réseau social et souvent de son statut social (cas des propriétaires coutumiers). La diversité des acteurs rend difficile l'accès à la propriété foncière car le foncier urbain fait l'objet de toutes les convoitises. En matière de gouvernance dans le cadre de la décentralisation, il n'y a pas eu de transfert à proprement parler mais seulement une déconcentration des services de l'État (pour preuve, il y a un agent du service des domaines et de celui de l'urbanisme dans toutes les mairies). La loi prévoit que tout propriétaire foncier (privé ou public) peut procéder à des lotissements en zone urbaine. L'État peut céder le terrain à une commune à titre gratuit si le programme le justifie dans le cadre des affectations aux services publics pour faire face aux demandes des citoyens. Dans la pratique, ce sont les autorités municipales qui attribuent les parcelles, même si cette attribution doit être approuvée par les autorités de tutelle (Gouverneur pour le District et les régions, préfet pour les Cercles, et sous-préfet pour les Communes). Mais chaque acteur développe sa stratégie pour se positionner sur le marché foncier 
urbain. Par exemple, de nombreux maires attribuent des parcelles sans l'accord des Domaines et du Cadastre, ni de l'Urbanisme. Il est de notoriété publique que la procédure d'attribution des parcelles par les maires est suspendue depuis 2000 par les autorités, mais dans la pratique, elle se poursuit clandestinement en attendant une ultérieure clarification des responsabilités des différents acteurs qui parait indispensable.

Les mutations ou changements qui en découlent sont la confusion quant au pouvoir respectifs des autorités communales, coutumières et l'autorité (Maire, Préfet, Sous-Préfet, Gouverneur). En théorie, le chef de village n'a plus le droit de vendre des terres sans l'avis préalable du maire et vice-versa. Le maire aussi doit avoir l'aval des autorités supérieures qui constituent les structures administratives de l'État. Une mutation majeure est le fait de confier le pouvoir foncier au maire avec la décentralisation ce qui va entrainer un dysfonctionnement dans le foncier. En effet, depuis quelques années la législation tente de s'imposer en droit foncier afin de faire reculer petit à petit le droit coutumier. Ce phénomène se concrétise avec l'augmentation des titres fonciers (TF). Une autre mutation de taille est qu'à partir de 1991, il y aura l'ouverture du marché foncier au secteur privé même si le marché foncier urbain reste marqué par son opacité, ce qui demeure une contrainte de taille en matière de sécurisation foncière surtout pour les couches pauvres. De même, les populations à faibles revenus ne participent pas au marché foncier formel car les parcelles viabilisées coûtent cher, et la procédure de régularisation du TF est longue et coûteuse. Ce manque de transparence dans le marché foncier a accéléré la création de quartiers spontanés (informels) qui se sont développés sur des terrains vendus par les propriétaires coutumiers sans infrastructures adéquates et généralement dans la périphérie de Bamako.

Les impacts de tout cet imbroglio en matière foncière sont la main mise sur le secteur de la part des responsables coutumiers, des agents de la haute administration, les édiles, les démarcheurs (coxers), les commerçants, les migrants, etc. L'analyse devient très difficile lorsque l'on se rend compte que ce sont les mêmes acteurs qui opèrent aussi dans le formel que dans l'informel. Un autre impact notable qui accentue la complexité du système foncier à Bamako réside dans l'existence d'une multitude de titres de propriété délivrés par les autorités (TF et LA). Une autre conséquence de ces mutations est l'absence de preuves juridiques, ce qui est à la base de beaucoup de conflits. Les Titres fonciers (TF) et les lettres d'attribution (LA) sont les voies officielles d'acquisition de parcelles ou de logement au Mali. Mais à côté de cette voie officielle coexiste et se maintient la voie traditionnelle. À côté de tout cela il y a les attestations de vente entre acquéreur et vendeur de parcelles qui même si elles impliquent la police ne l'engagent pas car elle ne cherche pas à vérifier la sincérité de l'opération. Ces attestations restent des actes de vente entre parties contractantes. Il Y a aussi des occupations sans titres ou 
«parcelles sans papier» Elles donnent droit à une occupation sans autorisation. Avant 1991, le Gouverneur était la seule autorité compétente en matière d'attribution de terrains.

Une autre conséquence de ces mutations est le changement de vocation des parcelles. Beaucoup de parcelles ont été détournées de leur but initial. Les exemples foisonnent à Bamako : Niamakoro-Sud dite «zone aéroportuaire » démolie en 1995 pour des besoins de sécurité, a été détournée. Également, la bande des 40m de Sabalibougou a connu d'autre utilisation que de recaser les déguerpis de Sabalibougou. Ces différentes violations perpétrées par certains responsables politiques véreux, des autorités administratives parfois, et d'autres individus influents, sont à l'origine de conflits fonciers à Bamako et ses environs. À cela il convient d'ajouter l'illégale occupation du lit du marigot de Niamakoro, les constructions illégales des berges du fleuve et le grignotage des ilots flottants sur le Niger à Bamako.

Après cette analyse de la compétition autour du marché foncier à Bamako, on peut affirmer que cette compétition relève bien de la théorie de «l'acteur stratégique» émise par Michel Crozier en 1970 selon laquelle étant donné qu'on ne peut considérer que le jeu des acteurs (ici acteurs de la gestion foncière) soit déterminé par la cohérence du système dans lequel ils s'inscrivent, ou par les contraintes environnementales, on doit chercher à comprendre comment se construisent les actions collectives à partir des comportements et des intérêts individuels parfois contradictoires (intérêts divergents des acteurs). La théorie du changement social de Guy Rocher élaborée en 1978 aussi trouve tout son sens dans le jeu des acteurs du foncier urbain à Bamako car il y a un « rapport de pouvoir » entre les acteurs qui n'ont ni les mêmes intérêts ni les mêmes stratégies. Aussi chaque acteur agit en fonction de son intérêt mais aussi en fonction de sa «capacité de négociation ».

Les facteurs du développement des tensions découlent du caractère litigieux des parcelles vendues par soit les propriétaires terriens, soit par l'État. Ces facteurs naissent aussi de la superposition des titres de propriété (la même parcelle cédée à plusieurs acquéreurs avec un titre de propriété). Ces titres sont généralement émis par les édiles (Maires). La localisation des parcelles dans des zones «non aedificandi » comme les lits des marigots, les berges du fleuve, les zones de servitude, etc. peut provenir aussi de la surenchère des coûts pratiqués par les coxers et promoteurs immobiliers.

\section{Conclusion}

Les autorités publiques du Mali (marie, ministère l'urbanisme, de l'habitat...), face à l'occupation anarchique et non autorisée de l'espace public en milieu urbain, mais aussi au refus d'obtempérer aux diverses injonctions émises, ont finalement recours à l'usage du bulldozer pour déguerpir « manu 
militari » les occupants des dits espaces (espace réservé ??). L’occupation non autorisée des espaces urbains (quel espace ?) est une stratégie des populations à faible revenus d'accéder à la propriété du sol en milieu urbain. La tactique ou politique du bulldozer est le dernier recours de l'autorité pour faire respecter ses injonctions en matière de gestion de l'espace public dans certaines zones à Bamako. L'autorité recourt à cette politique face au refus catégorique de certaines personnes d'obtempérer aux injonctions de l'autorité (Mairie, Gouverneur).

Cette technique doit être le dernier agissement qui intervient lorsque l'on aurait épuisé toutes les voies de recours prescrites. Cette tactique indique que « la force reste à la loi », mais elle fait des mécontents et aigris parmi les victimes qui deviennent des déguerpis et sont préoccupés par leur lendemain incertain dans l'espace urbain miné. Les conséquences de la tactique du bulldozer restent désarmantes mais renforcent la force publique. Les déguerpis qui résultent des démolitions deviennent des parias augmentant le nombre des sans-abris et le lot des mécontents qui constitue une bombe sociale en latence qui reste une menace non négligeable pour les gouvernants.

Cette étude empirique a permis de comprendre que la spéculation foncière qui a cours à Bamako est au centre d'une véritable économie urbaine informelle qui nourrit beaucoup d'hommes. De la même manière, après trois à quatre décennies de spéculation foncière (1970 à 2000), les réserves domaniales sont en voie d'épuisement, ce qui a pour conséquence de renchérir le prix de la parcelle et la diminution de ses dimensions pour les futurs acquéreurs. Ces deux précédents phénomènes débouchent sur une violence économique et sociale dans les zones à risque, ce qui accentue les troubles à l'ordre public et aux interpellations devant les autorités pour les parcelles vendues à deux ou trois clients, aux parcelles introuvables, à l'expropriation non signalée, bref au capharnaüm qui entoure le sol urbain.

Les hypothèses formulées au départ ont été toutes vérifiées.

La première hypothèse était que le recours à la politique du bulldozer par les gouvernants traduit l'échec des politiques foncière urbaines ce qui est amplement attesté par les résultats de cette étude. Également, les contraintes liées à l'accès à la propriété du sol urbain amènent les citadins aux revenus modestes à occuper illégalement l'espace public soit pour y habiter, soit pour mener des activités génératrices de revenus.

Le respect strict du contenu des documents de la planification urbaine aurait pu éviter l'occupation anarchique de l'espace public en milieu urbain. Ce qui a un effet, boomerang en ce sens que si les services techniques ne veillent pas scrupuleusement au respect de la réglementation en matière foncière, beaucoup de conflit vont y prendre corps qui vont attiser la contestation de plus en plus âpre de la gouvernance. Raison pour laquelle la Mairie doit servir de relais entre la population qu'elle est censée représenter et 
l'Etat détenteur du pouvoir de la «contrainte par corps » afin de faire « accepter » ses décisions .

Au bout du compte il est loisible de constater qu'en matière de gestion de l'espace urbain les autorités pratiquent la politique du sapeur-pompier plus que celle d'une gestion saine, c'est-à-dire, prévisionnelle de l'espace public en milieu urbain, principalement à Bamako.

À la lumière de ce qui précède, on peut affirmer que la politique du bulldozer, en dépit de ses limites, reste un outil de gestion «musclée » de l'occupation de l'espace public en milieu urbain.

\section{References :}

1. Ajay, K. \& Fanny, B. (2008). «Coincés dans les embouteillages, le transport urbain en Afrique ».

2. Aveline, A, (1995). «La boule foncière au Japon ».

3. Bakayoko, A. (1975). «La spéculation foncière sur la rive droite du fleuve Niger à Bamako : apparition et extension des quartiers sousintégrés »; Mémoire.

4. Bertrand (2015). « Du district au grand Bamako» (mali) : réserves foncières, gouvernance contestée », European Journal of Geography.

5. Bertrand (2015). «Mobilisations foncières à Bamako : des défis de la gouvernance à ceux de la citoyenneté »

6. Bouju (2009). « La malédiction, l’honneur et la spéculation. Principes historiques de la propriété foncière en Afrique de l'Ouest », Bulletin de l'APAD.

7. Bouju, J. (2003). «Invincibilités de la société civile, Espace public urbain, Société civile et gouvernance communale à Bobo-Dioulasso et Bamako (Commune 1 et 2) Rapport du programme de Recherche Urbaine pour le développement, Université de Provence, institut d'Etudes Africaines, P 148.

8. Bourdarias, F. (1999). «La ville mange la terre. Désordres fonciers aux confins de Bamako », _Journal des Anthropologues, mis en ligne le 1 Juin 2000, URL : http//Jda.revues.org /3072, consulté le 24 Février 2020-

9. Choplin, A. (2006). «Le foncier urbain en Afrique : entre informel et rationnel; l'exemple de Nouakchott (Mauritanie). In Annales de géographie.

10. Crozier, Michel, Friedberg, \& Erhard (1981). «L'acteur et le système », Editions du Seuil,1977,1981

11. Diarra, B. (2003). Caractérisation de l'environnement urbain par télédétection ; l'exemple de Bamako (Mali).

12. Diakité, Y. (2019). «Evolution des transports et de l'insécurité routière : cas du District de Bamako »,283 thèse. 
13. Deyoko, A. (1991). «Situation des quartiers spontanés à Bamako ; cas de Djikoroni Djénékabougou » in Maitriser le développement urbain en Afrique Subsaharienne, Paris, ORSTOM, pp 198-212.

14. Degbegnon, L. \& Toukourou, Y. (2016). « Expropriation foncière dans les grands projets d'intérêt national au Bénin : cas du projet de développement touristique de la «route des pêches » »

15. Fautras, M. (2016). « La terre entre racines, épargnes et spéculation : appropriations foncières et recompositions de l'espace rural de Regueb, Tunisie ». Thèse.

16. Franchette, S. (2011). «L'espace géographique ». Cairn.info.

17. Friedberg, E. (2006). « Jeux d'acteurs, Enjeux de pouvoirs »

18. Goislard, C. (1997). «Petites et moyennes villes d'Afrique noire».

19. Jalabert, G. (1971). «Spéculation foncière et urbanisation dans la banlieue de Toulouse », revue Géographique des Pyrénées et du Sudouest.

20. Keita, A. (2003). "Le phénomène des citadins paysans au Mali : stratégie de création d'exploitation agricole modern ou de spéculation foncière ? »

21. Keita, B. (2016). «Le marché foncier de Bamako et l'économie informelle » in Etudes Maliennes (ISH) N0 83/2016, P 132.

22. Loungou, S. (2014). «L'Espace politique », revue en ligne, consulté le. Novembre 2019

23. Traoré, K. (2019). " Mythes et réalités du recasement des populations déguerpies du district de Bamako : cas de la rive droite du fleuve Niger de 1960 à 2016 », thèse.

24. https/koulouba.com/spéculation foncière, consulté le...Novembre 2019 